\title{
Teleosemantics and Pushmi-Pullyu Representations
}

\author{
Marc Artiga \\ (forthcoming in Erkenntnis)
}

\begin{abstract}
One of the main tenets of current teleosemantic theories is that simple representations are Pushmi-Pullyu states, i.e. they carry descriptive and imperative content at the same time. In the paper I present an argument that shows that if we add this claim to the core tenets of teleosemantics, then (1) it entails that, necessarily, all representations are Pushmi-Pullyu states and (2) it undermines one of the main motivations for the Pushmi-Pullyu account.
\end{abstract}

\section{1- Introduction}

Teleosemantics is regarded as one of the most promising naturalistic approaches to intentionality. Since the first teleosemantic theories appeared in the mid 1980s (Papineau, 1984; Millikan, 1984) there has been an ongoing debate concerning several features of these theories. Nowadays, there are different teleosemantic theories on the market, but many current versions still heavily draw on Millikan's account (Millikan, 1984, 1993, 1995, 2004, 2005). In this paper I want to address one of the main claims of Millikanian teleosemantics, namely the existence of Pushmi-Pullyu representations. On Millikan's view, some representations (such as animal signals or the internal states of cognitively unsophisticated organisms) have both descriptive and imperative content. She calls such states 'Pushmi-Pullyu', after one of the animal character's in The Story of Dr. Doolittle, which has two heads at opposite sides of its body. Many people working within the teleosemantic framework have followed her in assuming that some representations carry descriptive and imperative content at the same time (McDonald and Papineau, 2006; Papineau, 2003b; Price, 2006, p. 216; Shea, 2007b; Matthen, 2006, p. 153).

In the paper, I present a central argument that has two striking consequences. On the one hand, I argue that adopting the Pushmi-Pullyu approach leads to an overattribution of descriptive and imperative content that teleosemanticists should reject. Secondly, the argument shows that the main motivation for embracing the Pushmi-Pullyu account is flawed. In a nutshell, my aim is to put forward two unwelcome consequences of endorsing the Pushmi-Pullyu theory within the standard teleosemantic approach. Whether that must lead to a rejection of the former or a revision of the latter is a question that I will not intend to address here.

The paper is structured in the following way. First, I will set up the core claims of mainstream teleosemantics, based on the notions of function, Normal explanation and sender-receiver structure. Secondly, I will present the Pushmi-Pullyu account and the main reason for endorsing it. Then, I will provide an argument that shows that (1) if the Pushmi-Pullyu account is right, then necessarily all representations are Pushmi-Pullyu and that (2) the motivation for adopting the Pushmi-Pullyu account is undermined. The argument intends to present two problematic consequences of adopting the Pushmi-Pullyu account within the standard teleosemantic approach. 


\section{2- Teleosemantics}

In this section, I will outline the core claims of the standard teleosemantic approach that compose the basic framework that is going to be assumed in the rest of the paper.

It is worth mentioning that there are different theories that are usually classified under the label 'teleosemantics', and they differ in significant respects. The particular version I will rely on roughly corresponds to Millikan's (1984) view, which probably is the most widespread version of the theory (e.g. Papineau, 2003a; Shea, 2007a; Godfrey-Smith, 1996). Furthermore, those that accept the Pushmi-Pullyu account also tend to endorse these core tenets of Millikanian Teleosemantics, so this is the version of the theory I will be focusing on.

\subsection{Function and Representation}

In order to present the core claims of teleosemantics, we need first to define three key notions: function, Normal explanation and sender-receiver structure.

\section{Function}

The relevant notion of function is the so called etiological. According to the etiological definition of function, a trait's function is the effect that explains why past traits of the same type have been selected for by some process (in the case of biological traits, this process is usually natural selection) (Wright, 1973; Millikan, 1984). On this view, functions are selected effects (Neander, 1991). For instance, the function of kidneys is to filter wastes from blood because this is the effect that explains why kidneys were selected for by natural selection. The appeal to functions is the distinctive feature of teleosemantic theories (Millikan, 2004), that distinguishes them from Causal Theories (Stampe, 1977; Dretske, 1981) and the Asymmetric Dependence Theory (Fodor, 1990; Margolis, 1998).

\section{Normal explanation}

A second important concept is that of a Normal explanation, which is defined by appealing to the notion of function. A Normal explanation (with a capital ' $\mathrm{N}$ ', to mark that it is a technical notion Millikan, 1984, ch. 2) is an explanation of how a particular trait has historically performed its function. More precisely, 'a Normal explanation is a preponderant explanation for those historical cases where a proper function was performed' (Millikan, 1984, p. 34). The Normal explanation of how a heart performed its function must mention the fact that it was supplied with blood, it was connected to the rest of the body through the right vessels, and so on. Similarly, Normal circumstances (or Normal conditions) are those circumstances that must be mentioned in a Normal explanation.

It is important to distinguish more detailed and least detailed Normal explanations. For instance, the least detailed Normal explanation of how a heart circulates blood must appeal to the oxygen supply, the presence of a closed circuit of blood vessels, and the regularity of electrical impulses sent to the heart (Millikan, 1984, p.33). A more detailed Normal explanation will tell us where the electrical impulses come from, the origin of the oxygen supply, and so on. At the very end, the complete Normal explanation must mention the fact that gravity remained constant, and any other feature that was present and had some relevance in Normal circumstances. As we will see, the key notion for teleosemantics is the least detailed Normal explanation.

Finally, it is worth pointing out that the Normal circumstances need not be the most common ones. The Normal circumstances for sperm to perform its function involves the presence of an 
ovum, since this is a condition that was present in those cases in which sperm performed its function, but obviously the presence of an ovum is not a statistically normal condition. Indeed, the occasions where there is an ovum to be fertilized are extremely rare. For a trait to evolve, it suffices if Normal circumstances obtain usually enough.

\section{Sender-Receiver}

The third important notion is that of a sender-receiver structure. According to teleosemantics, representations are states of affairs that stand between a sender and the receiver (Millikan, 1984, 1993; Godfrey-Smith, 1996, 2004; Shea, 2007a). To a first approximation, a sender (also called producer system) can be defined as a system that takes some external cues as inputs and generates the representation as output, whereas the receiver (or consumer system) is the system that takes the representation as input and generates certain activity as output (e.g. certain behavior).

With these three notions in mind, we are now in position to outline the key insights of the teleosemantic framework. A representation is a state that mediates between a producer and a consumer system. That is, a representation is a state produced by a certain device and interpreted or consumed by a second device. ${ }^{1}$ However, not any device can generate representations; representations are only produced within systems endowed with certain etiological functions. In other words, the producer and consumer system must have been selected for because they have historically performed certain tasks that are somehow useful for the organism(s). So, only when the sender-receiver structure is accompanied with the right etiological functions can we say a system is capable of producing representations.

In particular, the function of the sender is to help the consumer system to perform its own function by producing a state of affairs $\mathrm{R}$ (what we call the representation) when another state of affairs $\mathrm{S}$ obtains (the representatum). That is, the function of the system that generates representations is to help the interpreter by producing a state (the representation) that covaries with certain environmental circumstance that has some interest for the receiver (resources, predator,...). On the other hand, the function of the consumer system, which receives and interprets these representations, is to perform certain activities that (in Normal conditions) are only successful when the represented state of affairs obtains. The consumer system has been recruited because it performs some activities that are useful for the organism but the (least detailed) Normal explanation of how it has performed this function mentions the presence of the represented state of affairs obtains. Hence, while the function of the sender is to help the consumer by producing a representation that corresponds with certain world affairs, the receiver has the function of performing other activities, which can only be successfully carried out when there is a match between the representation and the represented affairs.

Let me illustrate this theory with a well-known example. Beavers splash the water with their tail so as to warn other beavers that danger is approaching. ${ }^{2}$ Here the sender is the beaver which splashes the water (or, rather, some mechanism within the beaver that is responsible for producing the splash); the receivers are the rest of beavers (or, rather, some mechanism within beavers that is

\footnotetext{
${ }^{1}$ The notion of 'interpretation' employed here can be defined in purely functional or causal terms: an interpreter (or consumer system) is the system that takes the representation as input and has the etiological function of yielding a certain activity (e.g. behavior) as output, So there is no reason to be suspicious about the naturalistic credentials of this notion. In any case, for the sake of the argument I will take for granted that a non-intentional characterization of the relevant teleosemantic framework is possible.

${ }^{2}$ A sender-receiver structure can be instantiated within a single organism or in different organisms. The example of alarm calling in beavers is a case of the second type, while the example of red-backed salamanders I suggest below (or the famous case of leopard frogs) is of the first type.
} 
responsible for the interpretation of the splash); finally, the representation is the water splash. This is a situation where the sender-receiver structure is instantiated; the function of the splashproducing-mechanism is to help other beavers to hide by producing a water splash when there is danger approaching. On the other hand, the function of the splash-interpreting-mechanism is to help the beaver to escape from danger. Now, the (least detailed) Normal explanation of how the splash-interpreting-mechanism achieves this result is by mentioning the fact that there was danger approaching. In other words, when there was no danger, escaping was a waste of time and energy; an explanation of why the splash-interpreting-mechanism has been selected for must mention the fact that there was a danger around. That is the condition that causally explains why having the interpreting mechanism was beneficial. Hence, according to teleosemantics, danger approacbing is what the splash-producing-mechanism is supposed to indicate.

\subsection{Core Teleosemantics}

Having sketched the three main concepts of teleosemantic and briefly explained how they yield a theory of representation, let us now define more formally the framework we will be working with.

First, we can define a sender-receiver structure taking into account what we saw in the last section:

SENDER-RECEIVER Necessarily, any two systems P (for Producer) and C (for Consumer) configure a sender receiver structure if, and only if:

1. $\mathrm{P}$ has the function of helping ${ }^{3} \mathrm{C}$ by producing state $\mathrm{R}$ when another state of affairs S obtains.

2. The function of $\mathrm{C}$ is to produce an effect (or set of effects) $\mathrm{E}$ (e.g. behavior). The least detailed Normal explanation for C's performance of $\mathrm{E}$ involves $\mathrm{S}$.

It is important to notice that SENDER-RECEIVER involves a modal claim: necessarily, if $\mathrm{P}$ and $\mathrm{C}$ satisfy 1 and 2 , then they instantiate a sender-receiver structure. Teleosemantics should be interpreted as involving a modal claim because it is naturalistic theory that seeks to spell out what representations and content really are. Teleosemantics intends to provide an account of what it is for a state to be a representation (and for this representation to have a certain content) in roughly the same sense chemistry is supposed to tell us what it is for something to be water (Millikan, 1993). So this theory is committed to the truth of certain modal claims. In particular, there has to be a necessary connection between satisfying the right-hand side of the definition and qualifying as a representation. What kind of modality is involved here (nomological, metaphysical or conceptual) is an important question that, unfortunately, I cannot address in this paper. ${ }^{4}$ For the time being, it suffices to see that teleosemantics is committed to certain strong modal claims holding true.

Once we have sketched a sender-receiver structure, one can define what representations are:

\footnotetext{
3 Again, I use the notion of 'helping' in order to simplify the definition. If anyone has naturalistic qualms about using this intentionally loaded notion, one could substitute condition 1 in Sender-Receiver for the following $1^{*}$ and $2^{*}$ :

$\left(1^{*}\right) \mathrm{P}$ and $\mathrm{C}$ have coevolved in such a way that a Normal condition for the proper performance of

P's function is the presence and Normal performance of C's function.

$\left(2^{*}\right) \mathrm{P}$ has the (relational) proper function of producing state $\mathrm{R}$ when another state of affairs $\mathrm{S}$ obtains.

${ }^{4}$ Let me just mention that it is usually thought to be (at least) metaphysical (Stoljar, 2010).
} 
REPRESENTATION Necessarily, a state $\mathrm{R}$ is a representation iff $\mathrm{R}$ stands between a sender and a receiver, which satisfy SENDER-RECEIVER.

Hence, whether a state is a representation depends on the existence of an adequate sender-receiver structure, which must be endowed with the right etiological functions. Accordingly, the content of a representation (what a representation is supposed to map onto) is determined by the functions of the producer and the consumer system. More precisely:

CONTENT Necessarily, R (descriptively) represents $\mathrm{S}$ iff:

1. $\mathrm{R}$ is a representation, in accordance with REPRESENTATION.

2. $\mathrm{S}$ is the state that must be mentioned in the least detailed Normal explanation of how R's consumer C performed its functions, in accordance with SENDER-RECEIVER.

Let me illustrate these last definitions with another example. Red-backed salamanders (Plethodon cinereus) have an internal mechanism that is sensitive to certain odors which are usually produced by predators, specially by eastern garter snakes (Thamnophis sirtalis) (Maerz et al. 2001). When this cue is present in the water surrounding the salamander, it significantly reduces its foraging behavior (Maerz et al. 2001). Now, there is an internal state (a signal) that is produced by the mechanism that is sensitive to the odor, which in turn is interpreted by another mechanism that reacts by reducing foraging activity. In this case, REPRESENTATION predicts that the internal sign is a representation, since this state stands between two systems with the right etiological functions. Similarly, it follows from CONTENT that the internal sign represents something like there is a predator around, since this is the state of affairs that must be mentioned in the least detailed Normal explanation of why reducing the foraging behavior was successful. Only when there was a predator around was this reduction of activity helpful; when there was no threat, the behavior was a lost chance for mating or ingesting food.

In short, teleosemantics claims that, necessarily, if two states $\mathrm{R}$ and $\mathrm{S}$ satisfy 1 and 2 in CONTENT, then $\mathrm{R}$ represents $\mathrm{S}$. More generally, we could say that the truthmaker for ' $\mathrm{R}$ represents $\mathrm{S}$ ' is the fact that the very complex causal and historical relation between states $\mathrm{R}$ and $\mathrm{S}$ and systems $\mathrm{P}$ and $\mathrm{C}$ defined in SENDER-RECEIVER, REPRESENTATION and CONTENT obtains. From now on, I will refer to these three theses as 'Core Teleosemantics'.

As I said earlier, I think Core Teleosemantics (or something very similar to it) could be accepted by many teleosemanticists. Nevertheless, it is worth mentioning that some philosophers have developed some views that differ in important respects from the key tenets discussed so far. In particular, defenders of so called 'input-based teleosemantics' (Neander, 2012) exclusively rely on the function of the producer system in order to determine the representational content. Thus, consumer systems do not play any essential role (e.g. Neander, 1995, 2012; Dretske, 1995). According to these views, the content of a representation $\mathrm{R}$ depends on what the R-producing mechanism is supposed to indicate, detect or co-vary with (McDonald and Papineau, 2006, pp. 7-8). But, similarly, input-based teleosemantic accounts are usually silent concerning the existence of Pushmi-Pullyu representations, probably because of their dismissal of consumer systems. So the arguments presented here only concern those teleological theories that require the existence of a sender-receiver structure, which happen to be the ones that also discuss the question of PushmiPullyu representations. 
Finally, notice that Core Teleosemantics is most intuitively understood as attributing only descriptive content to representations. From the analysis just given, it seems to follow that the representation tells the consumer system that a certain state of affairs obtains (the presence of a predator, food,...) in order to act accordingly. However, many teleosemanticists hold that an approach along these lines can also account for the existence of imperative content. This is the key claim I address in the remainder of the paper.

\section{3- Pushmi-Pullyu Representations}

Like many other approaches, teleosemantics is composed by a core set of claims (which roughly corresponds to SENDER-RECEIVER, REPRESENTATION and CONTENT) as well as other less central but also important assumptions that usually accompany the theory. The tenet I want to focus on is the claim that basic representations are what Millikan calls 'Pushmi-Pullyu' states. The basic idea of the Pushmi-Pullyu approach is that some representations have two kinds of representational content: on the one hand, they describe certain states of affairs and, at the same time, they tell the interpreter what to do. In short, Pushmi-Pullyu states are representations that have descriptive and imperative content. In terms of Anscombe (1957), they have a mind-to-world and also a world-tomind direction of fit. One of Millikan's favorite examples is the hen's call: when a hen finds food it makes a characteristic sound such that, on the one hand, tells the chicks that there is chicken food at certain place and, on the other, directs them to the food source (Evans and Evans, 1999). According to her, the hen's call means there is food at such and such location and, at the same time, tells the chicks go there and eat! (Millikan, 1995, p. 190; 2004, p. 80-1).

It is important to stress that, while a single state is said to carry both sorts of content, each representational content is determined differently. As suggested above, the state's descriptive content is determined by the state of affairs that must be mentioned in the least detailed Normal explanation of how the consumer performed its functions. In Millikan's (2005, p. 172) words, 'the descriptive representation's truth-conditions is a condition to which it adapts its interpreters or users in the service of their proper functions'. In the case depicted above, the hen's call descriptively represents there is food at such and such location because this is the state of affairs whose presence accounted for the success of the chick's behavior.

In contrast, the representation's directive content is determined by the effect that the representation is supposed to cause: 'a representation is directive when it has as a proper function to guide mechanisms that use it so that they can produce its satisfaction-condition' (Millikan, 2005, p. 171. Emphasis added). While a given mental state descriptively represents a condition that needs to obtain, it imperatively represents an effect that Normally ensues tokens of these representations.

Another example may be useful here. It is well known that honeybees have a very sophisticated communication system; when a working bee finds nectar, it returns to the hive and performs a figure-eight dance which varies in relation to the distance of the nectar found and the direction that a bee should take from the hive's entrance to the food resource taking the sun as the main reference. If the honeybee waggles very quickly in the direction opposite gravity, it leads other bees to fly (say) 800 meters to the sun, whereas if the dance consists of a short waggle oriented in a right angle to gravity, bees fly (say) 200 meters with the sun to their left. ${ }^{5}$ As most ethologists suggest (e.g. Gould, 1974) the so called 'waggle dance' is supposed to lead fellow bees to the source of nectar. Now, on the Pushmi-Pullyu framework we should say that the honeybee's dance indicates to

\footnotetext{
5 These particular contents are not empirically accurate.
} 
other bees the location of resources (there is nectar at 800 meters in the direction of the sun) and, at the same time, it tells its fellows fly off 800 meters in the direction of the sun and gather nectar!.

Summing up, according to the Pushmi-Pullyu account:

Pushmi-Pullyu Necessarily, if a representation $\mathrm{R}$ is supposed to make the consumer system $\mathrm{C}$ produce an effect $\mathrm{E}, \mathrm{R}$ imperatively represents $\mathrm{E}$.

PUSHMI-PULLYU seems to be in accordance with Millikan's (1995, p. 189) insights:

A representation is directive when it has a proper function to guide the mechanisms that use it so that they produce its satisfaction condition.

As I pointed out earlier, PUSHMI-PULlYU is accepted by many teleosemanticists (Millikan, 1984, 2004, 2005; Papineau, 2003b; Shea, 2007b, p.324; Matthen, 2006, p. 150; Price, 2006). Nevertheless, as I have presented the basic teleosemantic framework above, it is not obvious why a teleosemanticist should endorse it. Why should we believe that simple representations have a further content, besides the descriptive one? This is one of the main questions I would like to turn to now.

In general, Millikan (and all other teleosemanticists that follow her on that issue) argues by theory construction: she builds up a theory which is supposed to be supported by its empirical coverage and explanatory power relative to competing alternatives. Now, I think it is clear that in the case at hand there are serious alternatives; in particular, one could coherently hold what I will call the 'Simple Account', according to which teleosemantics only attributes descriptive content to representations. Such a Simple Account is obviously incompatible with PUSHMI-PULLYU but it is perfectly coherent with Core Teleosemantics. Thus, whether we should embrace PUSHMI-PULLYU depends on whether it provides any significant advantage over a Simple Account.

In what follows, I will try to bring forward and examine the main motivation in favor of PUSHMIPULLYU. The discussion is aimed at showing that the optimism teleosemanticists have usually expressed towards the Pushmi-Pullyu account may be unwarranted.

\subsection{Descriptive and Imperative content}

At this point, we have two alternative accounts, which are compatible with Core Teleosemantics but, obviously, mutually incompatible. On the one hand, the Pushmi-Pullyu Account (henceforth, PPA), which claims that simple representations have descriptive and imperative content at the same time. On the other, an approach that I call 'Simple Account', according to which simple representations are endowed only with descriptive content. Since teleosemanticists mostly argue by theory construction, one must assess whether, at the end of the day, the PPA has more explanatory power and less problems than the Simple Account or vice versa.

In this sense, probably the most important motivation for endorsing the Pushmi-Pullyu Account over a simple alternative is that the PPA seems to be able to account for the origin of imperative content. It is a platitude that some representations have imperative content (that is, a world-to-mind direction of fit). In fact, it seems obvious that some representations have only imperative content; let us call such states 'pure imperative representations'. Orders or desires are plausible examples of pure imperative representations. An utterance of 'Close the door!' is usually interpreted as directing the hearer to close the door. Examples of imperative representations and pure descriptive representations seem to be ubiquitous. 
Now, one might argue that by accepting PUSHMI-PULlYU and conceding that simple states have two kinds of content we are setting up the first step in order to explain how pure imperative and pure descriptive representations arise. Basically, the idea is that by assuming that simple representations have two kinds of content, we are likely to be in position to explain the origin of pure imperative representations (see Millikan, 2004, ch.15). So, the argument runs, the PPA account is preferable over the Simple Account.

I think this is a tempting argument, and the one that has led more people to find PPA attractive. However, in this section I will try to show that it is utterly misguided.

The argument I will put forward has two parts. First, I will argue that assuming PPA has a striking consequence, namely that necessarily all representations are Pushmi-Pullyu. This is what I call 'Overattribution':

OVERATTRIBUTION Necessarily, all representations are Pushmi-Pullyu representations.

I take that to be a highly problematic consequence. Perhaps some people might be willing to concede that indeed many representations that seemed to have only descriptive or imperative content in fact have two kinds of content. Some people could even accept that as a matter of fact all representations have two kinds of content. However, OVERATTRIBUTION claims something far stronger: necessarily, all representations have two kinds of content. The latter should undoubtedly be taken as an unwelcome consequence of the theory.

Now, as I have defined them, pure representations are representations that have only one kind of content (either descriptive or imperative), so if PPA entails that all representations are PushmiPullyu, it follows that PPA cannot account for the existence of pure imperative and pure descriptive representations. In other words, if the PPA entails OVERATTRIBUTION, then:

PURE REPRESENTAtions The PPA cannot explain how pure imperative and pure descriptive representations arise.

While Overattribution is a problem in its own, Pure Representations is supposed to undermine one of the main motivations for endorsing the PPA. If the PPA utterly fails to show that teleosemantics can account for the existence of pure representations, then it is surely unable to account for the origin of pure imperative representations. Hence, the main reason for preferring Pushmi-Pullyu Account over the Simple Account vanishes.

Crucially, notice that Overattribution and Pure Representations are claims that Millikan and other teleosemanticists want to deny. For instance, Millikan (2004) has argued that, paradigmatically, simple representations (such as alarm calls or mental states of cognitively unsophisticated organisms) are Pushmi-Pullyu states, but more cognitively sophisticated states, like some human mental states are pure descriptive or pure imperative representations. Similarly, Price (2001), Papineau (2003b) and Shea (2007b) seem to be willing to leave room for pure descriptive and pure imperative representations. They claim Pushmi-Pullyu representations are the most primitive forms of representation, and that at some point of the evolutionary scale, pure descriptive and pure imperative representations arise. The idea of Pushmi-Pullyu theorists, hence, is that at some level of complexity, evolutionary forces disentangle the two aspects of Pushmi-Pullyu states, in such a way that pure descriptive and pure imperative representations originate. Usually, they argue by pointing at many examples of representational structures that should be considered pure imperative and pure descriptive representations, like certain linguistic expressions or concepts. However, they fail to explain by means of which mechanisms both kinds of representational 
content are pulled apart. In what follows, I will try to argue that the existence of a mechanism that distinguishes the two kinds of content is incompatible with the key tenets of teleosemantics. In particular, I will show that Overattribution (and, hence, Pure Representations) follows from Core Teleosemantics and Pushmi-Pullyu. Consequently, if one accepts Pushmi-Pullyu and Core Teleosemantics, the descriptive and imperative content can not be disentangled.

Let me argue now why Core Teleosemantics and PUSHMI-PULlyU entail OvERATTRIBUTION.

\subsection{The Argument}

In a nutshell, the argument proceeds by showing that if a state qualifies as a representation, then it is a Pushmi-Pullyu state. So, we have to start by assuming that $\mathrm{R}$ is a representation and need to conclude that necessarily, $\mathrm{R}$ is a Pushmi-Pullyu representation.

First of all, REPRESENTATION claims that all representations are states that stand within a senderreceiver structure. In turn, from SENDER-RECEIVER, we know that a sender-receiver structure is composed of two systems, a sender and a receiver, which are endowed with certain etiological functions. The sender is supposed to help the consumer by producing a representation $\mathrm{R}$ when another states S obtains, and the function of the consumer is to produce other effects (e.g. behavior), which Normally are successfully carried out because the represented state of affairs obtains. This is the minimal structure required for a system to instantiate the sender-receiver framework.

\section{Descriptive content}

Now, notice that it follows from SENDER-RECEIVER that there is a state of affairs $S$ such that $S$ must be mentioned in the least detailed Normal explanation of how the consumer system performed its functions when $\mathrm{R}$ was tokened. If $\mathrm{R}$ is a representation, then (by REPRESENTATION) $\mathrm{R}$ is within a sender-receiver system and if $\mathrm{R}$ stands within a sender-receiver system, then there must be a state $S$ such that $S$ must figure in the Normal explanation of how the consumer performs its functions when $\mathrm{R}$ was tokened. So, for a state $\mathrm{R}$ to qualify as a representation, $\mathrm{R}$ has to fulfill REPRESENTATION and SENDER-RECEIVER, and if $\mathrm{R}$ satisfies both then there is a state $\mathrm{S}$ such that $S$ has to be mentioned in the Normal explanation of how the consumer performed its functions when $\mathrm{R}$ was tokened.

Secondly, according to CONTENT, if a state $\mathrm{S}$ must be mentioned in the least detailed Normal explanation of how the consumer system performed its functions when $\mathrm{R}$ was tokened, then $\mathrm{R}$ descriptively represents $S$. The claim that $R$ descriptively represents $S$ just means that $R$ is within a sender-receiver structure and $R$ is supposed to obtain when $S$ occurs. Consequently, for any representation $\mathrm{R}$, there is a state $\mathrm{S}$ such that $\mathrm{R}$ descriptively represents $\mathrm{S}$. Furthermore, since that results follow from what it is to be a representation, then this entailment has modal force. Necessarily, all representations have descriptive content.

\section{Imperative Content}

Similarly, it follows from SENDER-RECEIVER that the producer is supposed to help the consumer system. So, for any representation $\mathrm{R}, \mathrm{R}$ is supposed to bring the consumer system to have certain effects. The beaver's splash in the water is supposed to bring other beavers to escape; similarly, the hen's clucking is supposed to bring chicks to the hen's location and to ingest the food. So, if R is a representation, then it satisfies SENDER-RECEIVER, and if it fulfils SENDER-RECEIVER, then there is a consumer system $\mathrm{C}$ such that $\mathrm{R}$ is supposed to lead $\mathrm{C}$ to have certain effects. Thus, a representation is always supposed to lead the consumer system to do certain things. 
Now, Pushmi-PullyU states that if $\mathrm{R}$ is supposed to lead a consumer system $\mathrm{C}$ to produce an affect $\mathrm{E}$, then $\mathrm{R}$ imperatively represents $\mathrm{E}$. So if all representations are supposed to bring a consumer system to produce a certain E, and assuming that being supposed to have these effects on a consumer system suffices for a state to imperatively represent this effect (as PUSHMI-PULLYU claims), then all representations have imperative content. And, again, this claim has the same modal force as the previous ones. Therefore, necessarily, all representations have imperative content.

The conclusion of that reasoning is that all representations have descriptive and imperative content at the same time. In fact, since that follows from what being a representation is (and what content and sender-receiver structures essentially are), it follows that necessarily, all representations have descriptive and imperative content. That, I think, is a surprising consequence that Pushmi-Pullyu theorists should be uncomfortable with.

The argument can be expressed more clearly as follows:

(1) Necessarily, $R$ is a representation iff $R$ stands between a sender and a receiver, which satisfy SENDER-RECEIVER. (by REPRESENTATION)

(2) Necessarily, if $\mathrm{R}$ stands between a sender and a receiver, which satisfy SENDER-RECEIVER,

(a) There is a state of affairs $S$ that must be mentioned in the least detailed Normal explanation of how a R's consumer performs its functions when R is tokened.

(b) $\mathrm{R}$ is supposed to make the consumer system produce certain effects E. (by SENDERRECEIVER)

(3) Necessarily, if $\mathrm{R}$ satisfies 1 and 2a, $\mathrm{R}$ descriptively represents $\mathrm{S}$. (by CONTENT)

(4) Necessarily, if R satisfies 1 and 2b, R imperatively represents E. (by PUSHMI-PULLYU)

C) Necessarily, if $\mathrm{R}$ is a representation, then $\mathrm{R}$ descriptively represents $\mathrm{S}$ and imperatively represents E. (OVERATTRIBUTION)

In short, the argument shows that if a state $\mathrm{R}$ qualifies as a representation, then it stands between a sender and a receiver and, consequently, it is supposed to correlate with a state of affairs $\mathrm{S}$ and it is supposed to cause an effect $\mathrm{E}$ on a consumer. But if we also accept CONTENT and PUSHMIPULLYU, it straightforwardly follows that $\mathrm{R}$ has the two kinds of content. So by the mere definition of what representations are, it follows that they have the two kinds of content.

Therefore, Core Teleosemantics and PUSHMi-Pullyu entail Overattribution. And OVERATTRIBUTION is surely a thesis that teleosemanticists want to reject. As I pointed out earlier, one could assume that most representations have two kinds of content, or even that as a matter of fact all representations have them. However, the claim that necessarily, all representations are Pushmi-Pullyu is much harder to accept.

Furthermore, if PPA entails OvERATTRIBUTION, then PURE REPRESENTATIONS follows, that is, it has the result that the Pushmi-Pullyu Account cannot explain how pure imperative representations or pure descriptive representations arise, which was one of the main motivations for adopting PUSHMI-PULLYU in the first place. So the argument not only presents an unwelcome consequence of PUSHMI-PUllyU, but it also undermines one of the main motivations for preferring PUSHMIPULLYU over the Simple Account. 
Additionally, a nice feature of the argument I suggested is that it seems to explain why Millikan finds examples of Pushmi-Pullyu almost everywhere: all communication signals among nonhuman animals (2004, p. 157), perceptual states (2004, p. 159), many kinds of human conventions (2006), uses of linguistic expressions such as 'rude' or 'glorious' (1995, p. 198), performatives (1995, p.195) or even human intentions (1995, p. 192) are said to be Pushmi-Pullyu representations. If I am right, this result should not be surprising because, strictly speaking, Core Teleosemantics plus the Pushmi-Pullyu approach entails that necessarily all representations have both kinds of content.

Le me now consider some rejoinders the Pushmi-Pullyu theorists could try to pursue in order to avoid these striking consequences.

\subsection{Rejoinders}

I will consider three replies to my argument. The first one concerns Core Teleosemantics and the other two suggest slightly different versions of the Pushmi-Pullyu account. ${ }^{6}$

\subsection{Teleosemantics: Representations and Effects}

First of all, one might argue I have slightly misinterpreted the key tenets of teleosemantics. According to this objection, the argument presented above assumes that teleosemanticists are committed to a thesis that, in fact, they can reject. I will try to defend the accuracy of my description.

First, the objector may grant that teleosemantics accepts that, for any producer P and consumer C:

1) $P$ has to function to help $C$

2) $C$ has the function to do $E$.

Accordingly, teleosemantics seems to assume that for any representation $\mathrm{R}$ that $\mathrm{P}$ is supposed to produce:

3) $R$ is supposed to help $C$

However, premise $2 \mathrm{~b}$ in the argument claims something stronger, namely:

$\left.3^{*}\right) \mathrm{R}$ is supposed to make $\mathrm{C}$ perform $\mathrm{E}$.

Suppose the objector were right and teleosemantics were only committed to 1, 2 and 3. Since my argument requires $3^{*}$, which is a stronger premise, the argument presented above would not go through. Thus, the Pushmi-Pullyu theorist could avoid OveratTRIBUTION.

However, I think Teleosemantics is indeed committed to $3 *$. I will put forward three reasons for thinking that Teleosemantics must admit $3 *$ (and, of course, 3 as well).

First of all, it is not obvious that 3 and $3^{*}$ are really different claims. What it is for a representation to help a consumer system, if it is not to enable the consumer to do certain things? Remember that the notion of 'helping' is just a shorthand for the idea that the Normal condition for the performance of P's function involves the performance of C's function. So P helps C by contributing to carry out C's functions. Of course, there are still many questions open, for instance whether this set of effects of $\mathrm{C}$ must determined or not (I will consider this issue below). But, on

\footnotetext{
${ }^{6}$ I would like to thank an anonymous referee for pressing me on these objections.
} 
teleosemantics, 'helping a consumer' must always be interpreted as contributing to the fulfillment of C's functions.

Secondly, everyone in the debate assumes that, prima facie, the Pushmi-Pullyu theory and Teleosemantics are compatible. But the PPA (in any of its versions) claims that R imperatively represents whatever effects of $\mathrm{C} \mathrm{R}$ is supposed to elicit; so it assumes that (at least in many cases) $\mathrm{R}$ is supposed to make $\mathrm{C}$ have some effects $\mathrm{E}$. Therefore, the very plausibility of adding PPA to teleosemantics depends on the fact that teleosemantics accepts that, in many cases, $3^{*}$ holds.

Finally, there is a third and more interesting reason for thinking that Teleosemantics is in fact committed to $3^{*}$. This argument concerns descriptive content. What a given representation $\mathrm{R}$ is supposed to descriptively represent is determined by what the consumer needs in order to perform its functions (see CONTENT). So, in order for a representation to have a determinate descriptive content, there must be an effect (or set of effects) E that the representation is supposed to bring about. In other words, if there is no particular set of effects $\mathrm{E}$ of the consumer system that $\mathrm{R}$ is supposed to produce, then for any state of affairs $S$ and $S^{*}$, there is no fact of the matter as to whether R descriptively represents S or $\mathrm{S}^{*}$. Accordingly, a further reason for assuming that there must always be an effect or set of effects $\mathrm{E}$ a representation $\mathrm{R}$ is supposed to produce is that only if $\mathrm{R}$ is supposed to make $\mathrm{C}$ have some effects can we say it has a certain determined descriptive content.

In short, the last argument of this section can be expressed as follows: if $\mathrm{R}$ is a representation, it has descriptive content, and if it has descriptive content, there is a set of effects of $\mathrm{C}$ that $\mathrm{R}$ is supposed to help bring about. Finally, if there is a set of effects of $\mathrm{C}$ that $\mathrm{R}$ is supposed to help bring about, then $3 *$ is true. (This idea will be extended below). Therefore, I think Teleosemantics is committed to $3^{*}$ and the argument set up above stands.

\subsection{Coupled and Decoupled Representations}

Following the thread of this last objection, the Pushmi-Pullyu theorist may admit that perhaps all representations are supposed make the consumer have some effects, but nevertheless argue that there might still be a relevant distinction between different kinds of effects. In particular, one can suggest that representations that are supposed to elicit particular effects are Pushmi-Pullyu, while representations that are not supposed to produce any particular effects are purely descriptive.

A theoretical source for this objection is Sterelny's (2003) distinction between coupled and decoupled representations. Coupled representations are internal states of organisms in which there is a tight connection between the input and the output. For instance, coupled representations are the states in red-back salamanders that lead them to reduce foraging behavior when certain odor is present. At the other extreme, an example of decoupled representations are human cognitive states in the decision-making system, in which a single representation allows for many different decisions and actions by the agent. Human perceptual states are also usually thought to be decoupled representations (Matthen, 2006).

Following this distinction, it is usually claimed that only states that are supposed to elicit an automatic response on a consumer (i.e. coupled representations) are Pushmi-Pullyu. According to this proposal, when a consumer is in position to perform many different actions given a single representation, then we need not assume that the representation has imperative content. In other words: the less automatic the response elicited by a representation is, the less imperatively represented is that effect. The intuition here is that if there are many different things a consumer 
can do with a given representation, then there is no particular effect the representation is supposed to produce; in that case, the representation does not tell the consumer to do any particular thing. If at some level of cognitive complexity there is no particular effect a representation $\mathrm{R}$ is supposed to produce, then it seems $\mathrm{R}$ is not imperatively representing any particular effect.

Indeed, some quotes suggest that many teleosemanticists probably have this sort of view in mind:

When an internal state $\mathrm{C}$ ceases in this way to be devoted to any specific behavioral routines, it will cease to have any imperative content, and can be viewed as purely informational. (Papineau, 2003b, p. 88)

Representations of properties used for the purpose of detecting affording objects are not dedicated to particular practical purposes settled in advance. They are not pushmi-pullyu representations. They tell of the disposition of properties among various objects in the environment without yet saying what is to be done as a consequence. (Millikan, 2004, p. 174)

In a nutshell, this proposal suggests modifying PUSHMI-PULLYU so that only states that are supposed to elicit a particular effect qualify as Pushmi-Pullyu:

Coupled Pushmi-Pullyu Necessarily, if a representation $\mathrm{R}$ is supposed to make the consumer system $\mathrm{C}$ produce a particular effect (or a particular set of effects) E, R imperatively represents E.

Notice that in this definition I have added the possibility of $a$ set of effects being produced by a representation $\mathrm{R}$ (rather than only a single effect). The reason is that the reply based on the distinction between coupled and decoupled representations does turn on the question of how many effects are supposed to follow, but on whether there is a tight connection between input and output. For instance, suppose a rat is conditioned to press a lever when a light goes on. That would surely qualify as a Pushmi-Pullyu representation. Now, if the rat is supposed to press the lever only if the light is on and it is hungry, then there are two possible behaviors that the representation of a light being on may elicit, depending on the rat's hunger. Nevertheless, this scenario should still qualify as an instance of Pushmi-Pullyu representation (Millikan, 2004, p. 166-7). As it is usually understood, what is relevant is not how many behaviors are possible given $\mathrm{R}$, but whether the connection between input and output is more or less automatic or not.

Now, if one assumes COUPLED PUSHMi-Pullyu, then the main argument of the paper has to be modified. Accordingly, it only entails the following version of OVERATTRIBUTION:

COUPLED OVERATTRIBUTION Necessarily, if $\mathrm{R}$ is a representation that is supposed to make the consumer system $\mathrm{C}$ produce a particular effect (or a particular set of effects) $\mathrm{E}$, then $\mathrm{R}$ descriptively represents $\mathrm{S}$ and imperatively represents E.

In other words, only coupled representations are necessarily Pushmi-Pullyu. As a consequence, if one thinks that complex cognitive representations (like the ones involved in perception or the human decision-making system) are not supposed to produce particular effects, then one can block the conclusion that all representations are Pushmi-Pullyu.

However, I will argue this proposal fails to solve any of the two problems presented above. Coupled Pushmi-Pullyu also entails Pure Representations (which is the main motivation 
for any version of PUshmi-PullyU over the Simple View) and implies Overattribution. Let me explain why.

\section{Pure representations}

On the one hand, it is certainly true that while PPA has the consequence that all representations have two kinds of content, Coupled-PPA only entails that coupled representations have two kinds of content. However, as far as the theory goes, decoupled representations that satisfy Core Teleosemantics have only descriptive content, so Coupled-PPA does not explain in virtue of what process some representations are endowed with pure imperative content. In other words, if the PPA is restricted to coupled representations, then ex hypothesi the Pushmi-Pullyu theory does not contribute in any significant way to explain why decoupled representations have pure imperative content. So it does nothing to account for the existence of pure imperative representations. And since Coupled-PPA has the same explanatory power as the Simple Account concerning pure imperative and pure descriptive content had by some linguistic expressions or higher cognitive representations, we still lack a convincing argument for preferring it over the Simple Account. ${ }^{7}$

Indeed, not only does this theory, in its present form, fail to explain the existence of pure imperative representations in more complex systems; it also has the consequence that any representation that, according to the theory, has imperative content has also descriptive content. Here is the reason: suppose $\mathrm{R}$ is a representation with imperative content; then there is a consumer system that is supposed to elicit a particular effect $\mathrm{E}$ when confronted with $\mathrm{R}$ (by SENDERRECEIVER). But, of course, there will be some states of affairs that the consumer system requires in order to do E; the least detailed Normal explanation of C's performance of E will surely mention some circumstance $\mathrm{S}$. Since this is all that is required for a state $\mathrm{R}$ to descriptively represent $\mathrm{S}$ (see CONTENT), this proposal can not avoid the conclusion that all states with imperative content are also endowed with descriptive content. Again, PURE REPRESENTATION holds and, consequently, the main motivation for favoring the Pushmi-Pullyu Account over the Simple Account is undermined.

\section{Overattribution}

The most important question, however, is whether Coupled-PPA entails OvERATTRIBUTION. Concerning that particular issue, the first thing to notice is that the reply to my argument based on Coupled-PPA assumes that there are some representational states that are not supposed to elicit any particular response by the consumer (decoupled representations). However, prima facie, this case seems to be in tension with the main insights of teleosemantics. The standard cases teleosemantic theories have focused on involve a representation that makes the consumer produce some particular effect. Can teleosemantics explain the existence of decoupled representations at all? This is what Matthen (2006, p. 150) calls the 'Problem of Multiple Responses'.

Let me illustrate the most popular reply to the Problem of Multiple Responses with an example. Imagine that upon hearing a beaver tail splash, there is not any specific behavior beavers perform. Some beavers hide, others run away, others go to the place where the splash occurs (in order to attack the possible predator), others go to the burrow (to protect it) and some of them stop and reflect on what to do. We can also suppose that all these behaviors have somehow contributed to

\footnotetext{
7 PURE REPRESENTATIONS states that 'The PPA cannot explain how pure imperative and pure descriptive representations arise'. So, strictly speaking, Coupled-PPA does not entail PURE REPRESENTATIONS but something very close, that we could label PURE REPRESENTATIONS*: 'The Coupled-PPA cannot explain how pure imperative and pure descriptive representations arise'.
} 
the selection of the representational mechanism. That would seem to be a case of decoupled representation, since there seems to be no particular effect the representation $\mathrm{R}$ is supposed to produce. Now, in order to account for the descriptive content of this representation, teleosemanticists would probably suggest that, even if in this case there are many behaviors the splash prompts, the Normal condition for the performance of all these behaviors is the same state of affairs: the presence of a predator. Only when there was a predator around were all these different behaviors useful. Thus, the fact that many different behaviors are possible instead of one (the Problem of Multiple Responses) can be perfectly accommodated within Teleosemantics.

A place where this suggestion seems to be made:

If my belief that there is an umbrella in the hall closet is to help guide my decision-making and action-guiding apparatus (i.e. the belief's "interpreter") such that it serves its function of helping fulfill my desires, for example my desire to keep off the rain, then there needs to be an umbrella in the hall closet. If it is to help me make a correct inference concerning, for example, whether or not Susan returned my umbrella, one that yields this truth not by accident but in accordance with the good design of my cognitive systems, still there needs to be an umbrella in the hall closet - and so forth. (Millikan, 2005, p. 172)

I think this account of descriptive content is quite satisfactory. However, this response to the Problem of Multiple Responses shows that the Pushmi-Pullyu accounts falls prey to my argument. Let me explain.

If a state qualifies as a representation, then it has some descriptive content. According to Core Teleosemantics, this descriptive content will be determined by the state that must be mentioned in the least detailed Normal explanation of how the consumer performed certain functions (see CONTENT). So R's descriptive content depends on the Normal conditions for performance of a set of functions $\mathrm{E}$. Now, that means that there will be a set of effects $\mathrm{E}$ (which might be very broad) of the consumer $\mathrm{C}$ that is being considered as relevant for the determination of R's descriptive content. Surely, not any possible behavior elicited by $\mathrm{R}$ is relevant for content determination. Thus, there is a set of effects that $\mathrm{R}$ is supposed to produce, and my argument still holds. Therefore, after all teleosemantics is committed to the existence of a set of effects the representation is supposed to cause. And, again, the reason is that if a representation has descriptive content, then there are some effects that $\mathrm{C}$ is supposed to perform. And since the determination of descriptive content requires the specification of a set of relevant effects, the argument I presented above still goes through.

In other words; the descriptive content of $\mathrm{R}$ is the state of affairs that constitutes the Normal condition for the performance of a certain set of behaviors (see CONTENT). But for a state of affairs to be the Normal condition for the performance of certain behaviors, we must pick up a definite set of behaviors that were somehow relevant in the selection of this state. Otherwise, we would lack a criterion for selecting a state of affairs as being the Normal condition rather than any other state of affairs. But if that is the case, there is a definite set of behaviors that this representation Normally generates. So this is no objection to my argument.

In fact, what I am saying here seems to be in accordance with certain ideas expressed by teleosemanticists. For instance, in some places Millikan seems to suggest that decoupled 
representations can be Pushmi-Pullyu states. In particular, she argues that percepts are also PushmiPullyu, even if there are many different behaviors perceptual representations can lead to:

Think of perceptual representations simply as states of the organism that vary directly according to certain variations in the distal environment. The perceived layout of one's distal environment is, first, a representation of how things out there are arranged - a descriptive representation. It is also a representation of possible ways of moving within that environment: ways of passing through, ways of climbing up, paths to walk on, graspable things, angles from which to grasp them, and so forth. Variations in the layout correspond to variations in possible projects, and in the paths of motion needed to achieve them. The representation of a possibility for action is a directive representation. (Millikan, 1995, p. 191)

That suggests that the fact that a representation is to some extent 'decoupled' does not show that there is no set of effects $\mathrm{R}$ is supposed elicit on the consumer. So the argument leading to OVERATTRIBUTION holds. ${ }^{8}$

Note that I need not deny that the distinction between coupled and decoupled representations is relevant in many respects. For instance, it might well be that the theory predicts that the kind of imperative content of coupled representations is much more determined that the kind of imperative content of decoupled representations. My argument just requires that there is some effect or set of effects that the representation is supposed to elicit.

There is, however, a final reply I would like to consider.

\subsection{Basic representations}

Some people might think my last reply has somehow misinterpreted the objection. Thus, before concluding, I would like to address a different worry that might be suggested by the previous considerations.

This final objection is more general and can be presented as follows. There are surely many differences between basic representations (states in bacteria, frog's mental states, etc.) and nonbasic ones: basic representations usually generate behaviors (rather than decisions or other internal states), some of these representations probably are unconscious, some represent concrete rather than abstract entities, and so on. Why can the Pushmi-Pullyu theorist not just pick up one of these properties as the one that marks the difference between Pushmi-Pullyu states and the rest? Basically, the idea is to take whatever feature $\mathrm{F}$ distinguishes basic from non-basic representations and claim that only F-representations are Pushmi-Pullyu. Indeed, one can interpret the objection of Coupled-PPA as a particular version of this objection.

Accordingly, one could modify PUSHMI-PULLYU so as to get following ReSTRICTED PUSHMIPULLYU:

RESTRICTED PUSHMI-PUllyU Necessarily, if a basic representation $R$ (i.e. a representation instantiating $\mathrm{F}$ ) is supposed to lead a consumer system $\mathrm{C}$ to have certain effect $\mathrm{E}, \mathrm{R}$ imperatively represents $\mathrm{E}$.

\footnotetext{
${ }^{8}$ Indeed, remember that, if the PPA entails OveratTRIBUTION, then PURE REPRESENTATIONS holds. This is a more indirect way of showing that Coupled-PPA entails PURE REPRESENTATIONS (or PURE REPRESENTATION*, see footnote 7).
} 
If PUSHMI-PULLYU is modified in that way, then premise 4 of the argument should also have to be restricted to basic representations. As a consequence, instead of OVERATTRIBUTION, we can only conclude RESTRICTED OVERATTRIBUTION:

RESTRICTED OVERATTRIBUTION Necessarily, if $\mathrm{R}$ is a basic representation, then $\mathrm{R}$ descriptively represents $\mathrm{S}$ and imperatively represents $\mathrm{E}$.

Of course, this proposal needs to be complemented with an account of what it is to be a basic representation (i.e. what is the crucial property F). A proposal might be that basic representations always elicit a single effect (e.g. throwing the tongue out) rather than a set of effects, or that basic representations are those that make the consumer produce some action (rather than a decision). Many other proposals are possible.

Unfortunately, I think Restricted-PPA cannot avoid any of the problems of PPA. First of all, both entail that teleosemantics cannot account for the origin of pure imperative representations (PURE REPRESENTATIONS), so the main motivation of the theory is undermined. Since the reasons here are exactly the same I presented in the section on Decoupled-PPA, I will not repeat them.

More interestingly, Restricted-PP is also very likely to entail that necessarily, all representations are Pushmi-Pullyu (OvERATTRIBUtion). So I do not think Restricted-PPA offers any significant advantage over PPA or the Simple View. This is a claim that needs to be argued for.

\section{Overattribution}

There are two features of standard teleosemantics that, when put together, makes it difficult for Restricted-PPA to avoid OVERATTRIBUTION.

First, it is important to keep in mind that teleosemantics is a naturalistic theory that tries to describe an objective process by means of which some states are endowed with representational content (as we saw, this is the reason it is committed to the truth of certain strong modal claims). It assumes there is some observer-independent feature that accounts for the semantic properties of representational states. So one cannot merely stipulate that a certain sort of entities have imperative content; one needs to find an objective property that explains why there is a distinction between Pushmi-Pullyu representations and the rest.

Secondly, it is commonly assumed in teleosemantics that there is a continuum between basic and less basic representations. Of course, there are many differences between representations of bacteria or the frog's mental states on the one hand, and human beliefs or perceptual states on the other (Millikan, 1984, ch. 7; Papineau, 2003b). Nevertheless, teleosemanticists usually claim there is a relevant sense in which all of them are representations. Indeed, teleosemantics is supposed to capture the aspect that all of them share. For instance, Millikan (2004, p. 158) writes:

The bottom-most level of inner Pushmi-Pullyu signs is ubiquitously exemplified, not merely in neural matter, but in many chemical messengers found in the body tissues and the circulatory systems of animals. (...) To suggest that genuine intentionality, genuine aboutness, with the possibility of misrepresentation, actually occurs at this level may at first seem far-fetched. But the idea is that there is intentionality here in the sort of way zero is a number. By treating such simple signals as intentional signs, just as by treating zero as a number, we will be able to examine their relations to various successors, and see the continuity between them and their more sophisticated relatives. 
These two features of teleosemantics show that it is not enough to find a property $\mathrm{F}$ that distinguishes basic from non-basic representations. This property (1) must be observer-independent and (2) must explain why there is a difference in content between representations. It is not obvious that any of the previous candidates can explain this difference. For instance, even if basic states usually represent concrete entities and more complex states can represent abstract entities, that property does not seem to mark a significant line between Pushmi-Pullyu representations and the rest because there is no reason for thinking this particular difference would establish a difference in content. Similarly, in responding the previous objection, I showed that the fact that some representations are decoupled does not constitute a significant difference either. Thus, the onus is on the side of the Pushmi-Pullyu Theorist. Unless an objective property is found that clearly distinguishes two kinds of representations (basic from non-basic representations) and grounds a distinction between different kinds of content, the claim that simple representations are PushmiPullyu will probably entail that all of them are. The general hypothesis that there is a continuum between all kinds of representations seems to lead to that conclusion.

A caveat is important here. Notice that this argument is perfectly compatible with many distinctions between basic and more complex representations. For instance, in contrast to basic representations, some complex representations exhibit productivity, abide by the compositionality principle or represent different kinds of facts. In that respect, there is a vast literature on several features that draw significant lines between representations of different kinds (e.g. Bermudez, 2003). But, given the standard assumption of an underlying continuum among representational systems, in order to block the previous argument one must find a property $\mathrm{F}$ that distinguishes basic and complex representations such that it can ground a distinction between kinds of content had by the representations. In other words, the key property we are looking for not only needs to distinguish basic from less basic representations; it also has to explain why we need to suppose there is a distinction in content between them. We saw that the distinction between coupled and decoupled does not do the trick. The challenge for the Pushmi-Pullyu theorist is to find a successful candidate.

In fact, there is an additional reason for thinking there is no property $\mathrm{F}$ that can play this role. Some teleosemanticists like Millikan think that some cognitively sophisticated states and linguistic expressions are also Pushmi-Pullyu states (e.g. 'No Johnny, we don't eat peas with out fingers' Millikan, 2004, p. 157). So the property we are looking for in order to avoid OvERATTRIBUTION has to be a real feature that distinguishes these complex representations that are Pushmi-Pullyu from the rest of complex representations that are pure representations. That shows that the task of finding out a property $\mathrm{F}$ that can play this role may be extremely difficult.

Therefore, if the reasoning suggested here is on the right track, Restricted-PPA entails PPA and hence, necessarily all representations $\mathrm{R}$ imperatively represent an effect. OVERATTRIBUTION is back.

\section{Conclusion}

In this paper, I have presented an argument that shows that accepting Core Teleosemantics and any version of PUSHMI-PULlyu (1) undermines one of the main motivations for embracing the Pushmi-Pullyu Account (2) entails that, necessarily, all representations are Pushmi-Pullyu. Whether the arguments provided in this paper utterly undermine the Pushmi-Pullyu Account or, alternatively, suggest a modification of some of the central thesis that compose Core Teleosemantics is something that I have not attempted to address here. I hope the paper has at least been able to shed some light on one of the most obscure notions of current teleosemantic proposals. 


\section{Acknowledgments}

I would like to thank Agustin Vicente, Brian Leahy, Manolo Martinez, David Pineda, an anonymous referee and the audience of the 6th Latin Meeting for Analytic Philosophy for helpful comments and incisive criticisms. This work was supported by the scholarhsip BES-2008-005255 from the Spanish Ministry of Science and Innovation (MICINN), the Research Projects 'The Naturalization of Subjectivity' (ref. FFI2010-15717), 'Modal Aspects of Materialist Realism' (ref. HUM2007-61108) and Consolider-Ingenio project CSD2009-00056.

\section{References}

Anscombe, G. E., (1957) Intention, Harvard University Press

Bermudez, J. L. (2003) Thinking Without Words, Oxford University Press

Dretske, F. (1981) Knowledge and the Flow of Information. The MIT Press.

Dretske, F. (1995) Naturalizing the Mind, The MIT Press.

Evans, C., Evans, L. (1999) Chicken food Calls are Functionally Referential, Animal Behavior. Vol 58(2): 307-319

Fodor, J., (1990) A Theory of Content and Other Essays. The MIT Press.

Gould, J.L. (1974) Honeybee communication. Nature 253: 300-01

Godfrey-Smith, P. (1996) Complexity and the Function of Mind in Nature, Cambridge University Press, Cambridge

Godfrey-Smith, P. (2004) Mental Representation, Naturalism and Teleosemantics. In Papineau D. and McDonald, G. (2004) Teleosemantics: New Philosophical Essays. Oxford, Oxford University Press.

Margolis, E., (1998) How to acquire a concept. Mind and Language, 13(3): 347-369.

Matthen, M. (2006) Teleosemantics and the Consumer, in McDonald, G. \& Papineau, D. (ed.) Teleosemantics, Oxford University Press.

Maerz, J., Panebianco, N. L., Madison, D. M. (2001) Effects of Predator Chemical Cues and Behavioral Biorhythms on Foraging Activity of Terrestrial Salamanders. Journal of Chemical Ecology. Vol. 27 (7): 1333-1344

MacDonald, G., Papineau, D, (2006) Prospects and Problems for Teleosemantics. In Papineau, D, MacDonald, G. (2006), Teleosemantics, Blackwell

Millikan, R. (1984) Language, Thought and Other Biological Categories. Cambridge (Mass.), MIT Press

Millikan, R. (1993) White Queen Psychology and Other Essays for Alice. Cambridge (Mass.), MIT Press

Millikan, R. (1995) Pushmi-Pullyu Representations, Philosophical Perspectives, 9:185-200. 
Millikan, R. (2004) Varieties of Meaning, Cambridge (Mass.), MIT Press

Millikan, R. (2005) Pushmi-Pullyu Representations. In Millikan, R. (2005) A Biological Model, Cambridge (Mass.), MIT Press

K. Neander (1991) Functions as Selected Effects: The Conceptual Analyst's Defence, Philosophy of Science, 58:168-184, 1991.

Neander, K. (1995) Misrepresenting and Malfunctioning. Philosophical Studies 79 (2):109-41

Neander, K. (2012) Teleological Theories of Mental Content, Stanford Encyclopedia of Philosophy

Papineau, D. (1984) Representation and Explanation, Philosophy of Science, Vol. 51: 550-72

Papineau, D. (1998) Teleosemantics and Indeterminacy, Australasian Journal of Philosophy, 76 (1): $1-14$.

Papineau, D. (1993) Philosophical Naturalism, Blackwell

Papineau, D. (2003a) Is Representation Rife?, Ratio, 16 (2): 107-123

Papineau, D. (2003b) The Roots of Reason, Oxford, Oxford University Press

Price, C. (2001) Functions in Mind. Oxford, Oxford University Press

Price, C. (2006) Fearing Fluffy: The Content of an Emotional Appraisal. In Papineau, D, MacDonald, G. (Eds.) Teleosemantics, Oxford University Press, Oxford.

Shea, N. (2007a) Consumers need Information: Supplementing Teleosemantics with an Input condition. Philosophy and Phenomenological Research, 75(2): 404-435

Shea, N. (2007b) Representation in the genome and in other inheritance systems, Biology \& Philosophy, 22 (3): 313-331

Stampe, D. (1977) Towards a Causal Theory of Linguistic Representation, Midwest Studies in Philosophy, 2(42): 63

Stoljar, D. (2010) Physicalism, Taylor and Francis.

Stegmann, U. (2009) A Consumer-Based Teleosemantics for Animal Signals. Philosophy of Science, $76(5)$

Sterelny, K. (2003) Thought in a Hostile World: The Evolution of Human Cognition, Blackwell Publishing

Wright, L. (1973) Functions. Philosophical Review, 82:139-168. 